\title{
Efficient ABC Algorithm for Dynamic Path Planning
}

\author{
Priyanka Goel \\ Department of Computer Science \& Information \\ Technology, \\ Moradabad Institute of Technology \\ Ram Ganga Vihar, Phase-II \\ Moradabad, Uttar Pradesh, India
}

\author{
Devendra Singh \\ School of Computer Engineering and Applications, \\ Department of Computer Science \\ IFTM University \\ Lodhipur Rajput, Delhi Road (NH-24) \\ Moradabad, Uttar Pradesh, India
}

\begin{abstract}
The path planning of mobile robot is an important issue in the field of robotics. Many algorithms have been designed to solve the path planning problem, including classical as well as intelligent approaches. The main aim of path planning is to construct collision free path from a specified start position to the target position. Moreover, the path should be optimal in some context such as distance, time or processing. This paper presents an efficient algorithm which is a variation of artificial bee colony algorithm. The environment modelled is in the form of grid consisting of obstacles. The algorithm works on two problems- the first problem is to find collision free path in the presence of static obstacles and the second problem is to determine shortest collision free path in the presence of dynamic obstacles.
\end{abstract}

\section{Keywords}

Path planning, efficient, dynamic, optimal

\section{INTRODUCTION}

Robots are now serving almost every field ranging from domestic assistance to robot-assisted surgery and are taking place of human beings especially for accomplishing various hazardous tasks such as bomb diffusion, manufacturing processes, mining etc. Earlier, the path planning problem used to be studied in the field of robotics but today it has gained importance in other fields such as simulation, computer graphics, geographic information systems, very large scale integrated (VLSI) designing and games. This field remains the core area in many of the robotic applications such as autonomous vehicle designing and perceptive systems. The scope of path planning can be categorized into various streams depending on the problem statement, environment modeled, and types of sensor [1]. The path planning problem can be solved to attain various optimization goals such as minimizing distance travelled, maintaining smooth trajectory or satisfying the clearance requirements [2]. Path planning problem can be solved off-line or off-line. In the first case, the environment and the positions of obstacles are known before determining the feasible path and global view of the environment is analyzed beforehand. In the latter case, local view of the environment and the obstacles is obtained and the path planning is performed using the local information obtained with the help of some sensing device. The traditional algorithms used for path planning have certain limitations such as low search efficiency, complex search paths, large convergence time and trap into local maxima[3-8]. So the interest of researchers switched to intelligent algorithms which are proven to be much better than classical approaches. Many philosophers and researchers have been working for centuries on the problem of social choice. The main interest is to study how different creatures in nature work together in a group to accomplish a task. A branch of nature inspired algorithms which are known as swarm intelligence is focused on insect behavior so as to develop some meta-heuristics which can imitate the way insects used to solve their problems [10-16]. Swarm intelligence consists of many algorithms such as ant colony optimization, particle swarm optimization, wasp nets and fish schools.

Swarm intelligence approaches imitate nature's way to find optimal solution. These approaches generate population of solutions for every iteration. All population based approaches follow a strategy that generates variations in the solutions. This strategy may employ greedy criterion or non-greedy criterion to decide which solution to retain. This method selects a solution which increases the value of objective function (assuming the objective function is maximization function).

\section{PROBLEM FORMULATION}

The proposed algorithm works on two-dimensional environment which is modelled in the form of grid. The grid is discretized in the way that each cell (called as node) is assigned a number so as to simplify the representation of the path. The source and destination positions are chosen by the user. The user is free to place the static and dynamic obstacles and their number is determined by the grid size. The robot is considered to have constant speed in order to determine shortest path [17]. The proposed work in this paper considers following assumptions:

i. Static obstacles have pre specified shape.

ii. Moving obstacles have same speed and their trajectories are in linear direction.

iii. The robot has been considered as a point object to keep the minimum safety distance from the robot to the obstacles.

The proposed work aims to determine the shortest collision free path from the specified source position to the destination position. Each solution generated is termed as path and each path is composed of nodes. The number of nodes in a path is kept fixed for simplification purposes.

\section{BEES' BEHAVIOUR IN NATURE}

There are many examples which show perfect coordination and decision making in an animal group. A striking example is the swarm of bees which consists of about 10,000 honey bees. The process involves finding a better nesting site which is performed by dozens of bees together. This process has been studied widely, investigated and examined thoroughly and the conclusion drawn is that this group intelligence is a product of disagreement and contest and not consensus or 
compromise. Bees dwell in colonies that extend over very long distances. They explore multiple directions in order to exploit food sources. The flower patches containing large amount of nectar that can be extracted with minimum difficulty are visited by most of the bees while the nectar sources with less amount of nectar are discarded. The foraging process begins by scout bees which are sent to collect nectar. The search starts randomly by the scout bees from one patch to another. On reaching the hive, scout bees, deposit the amount collected and go to the dance floor to perform the waggle' dance.

This type of dance is the sole medium of communicating the information about the most promising nectar source to all other bees in the hive. This mysterious dance contains three pieces of information about flower patch: direction in which it is found, distance from the hive and the quality of nectar. When the food source exists at a distance less than 50 meters, then the scout bees perform round dance. On the other hand, if the food source exists at a distance greater than 50 meters, then the bees perform waggle dance.

After watching the waggle dance, unemployed bees (onlooker bees) follow the scout bee to the promising nectar source to collect nectar. This process helps all the bees to collect nectar in efficient and faster way. The quality of food source is continuously monitored by the bees so as to propagate this information in the next waggle dance. Thus the natural bee system consists of following components:

i. Food sources: The value of food source depends on certain parameters such as proximity of the source from the hive, richness of nectar and ease of extracting nectar.

ii. Foragers: If a bee has no knowledge about the food source initially then it is said to be an unemployed forager. It may start searching according the following possibilities as:

a. Scout bee: If a bee starts searching randomly for a food source without any prior knowledge, then it will be a scout bee. The percentage varies from $5 \%$ to 30 $\%$ depending on the food source information in the hive.

b. Onlooker bee: If a bee watches waggle dance done by another bee then it starts searching according to the information obtained from waggle dance.

iii. Employed foragers: When the onlooker bee finds and exploits the food source then it becomes employed forager who memorizes the food location. After bringing the nectar to the hive, there are three possibilities for an employed bee to do:

a. If the nectar amount decreased to low level, then the employed bee rejects the food source and becomes an unemployed bee.

b. If the nectar amount is up to sufficient amount then it starts exploiting the food source itself without informing to the other bees.

c. Otherwise it goes to the dance floor of the hive and performs waggle dance in order to inform other bees about the food source.

These specialized bees try to maximize nectar amount in their hive using efficient division of labor and self-organization. Artificial Bee Colony optimization is a population based optimization technique which was first proposed by Karaboga in 2005. It is an algorithm which imitates the foraging behavior of bees to solve unconstrained optimization problems with continuous valued domains. Half of the colony bees become employed bees and the other half comprises of onlooker bees.

\section{PROPOSED WORK}

The proposed algorithm for path planning in dynamic environment is based on artificial bee colony algorithm hybridized with some new operators in order to obtain collision-free efficient paths. The path planning is performed in two stages- path planning in static environment and path planning in dynamic environment. Figure 1 shows a snapshot depicting our grid environment in which the white grids indicate the static obstacles and the line segments indicate the trajectory of dynamic obstacles.

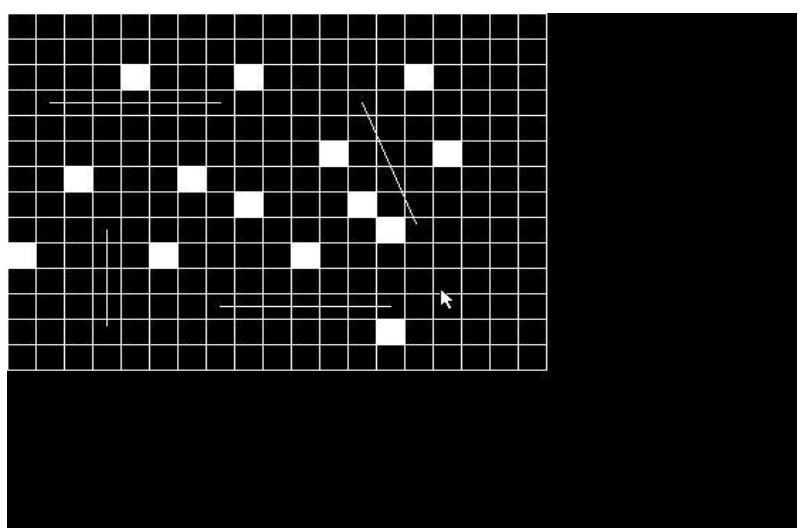

Fig 1: Grid environment used in simulation

\subsection{Path Planning in Static Environment}

The algorithm initializes the population size of the bees and the value of maximum cycles. The population size consists of an equal number of employed bees and onlooker bees. Assign the number of food sources as:

FoodNum= Population Size/2

FoodNum paths are generated randomly in the initial stage The objective function is calculated for each generated path. The objective function value is equal to the distance between two corresponding nodes and a defined penalty is added in it for collision with static obstacle. For each path generated path, fitness function is calculated according to the equation:

fitness $_{i}=1 / \mathrm{F}_{\mathrm{i}}$

where $F_{i}$ is the objective function value for path i. Based on the fitness value, employed bees are send to improve the path further. The nodes containing the obstacles in the path are the source of food which needs to be improved. In order to improve this path, new nodes are generated using the 8neighbour rule. Then new path is constructed replacing the existing node and objective function is calculated again. If the new path is better than the existing path then the path is updated otherwise trial value of the path is incremented by 1.This process continues for all food sources. Two new operators nodeDelete and segmentRepair have been used to obtain the shortest final paths If the trial value exceeds a certain limit, then that path is abandoned and its employed bee becomes a scout bee. The employed bees whose paths are abandoned become scout bees and start searching a new path randomly and the whole process is carried out until the stopping criterion is met.

4.2 Path Planning in Dynamic Environment 
All the paths generated in stage 1 are arranged in the decreasing order of fitness value and the onlooker bees are assigned to each path accordingly. Now the cost of best paths is evaluated by considering the dynamic obstacles. Each onlooker bee attempts to improve the path by generating random values and assigning fitness value to each path generated. If the trial value for a particular path exceeds the limit then that path is abandoned and random search for a new path is started.

\subsection{Operators Hybridized with Artificial Bee Colony Algorithm}

The operator nodeDelete is applied to both feasible and infeasible path. This operator randomly choose one node, check its two adjacent nodes, and connected segments, if the deletion of the chosen node is beneficial (turn the infeasible to the feasible or reduce the cost), then it deletes the node as shown in Figure 2.

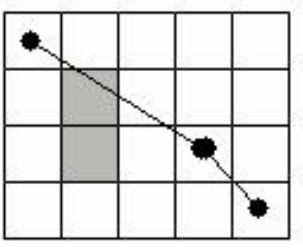

(a)

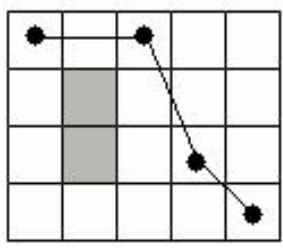

(b)
Figure 2: Working of nodeDelete operator. (a) shows the scenario where some unnecessary nodes are present in path (b) shows the path after applying nodeDelete operator

Another operator segmentRepair is used to repair an infeasible line segment by inserting a suitable node between the two nodes of the segment as shown in figure 3. Again, to locate a best node, similar local search is applied in the all of neighboring grids of the intersected obstacle.

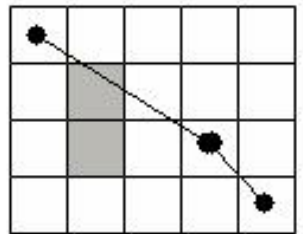

(a)

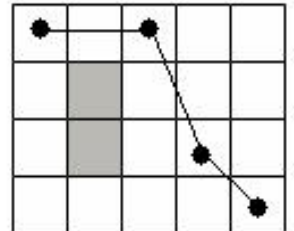

(b)
Figure 3: Working of segmentRepair operator. (a) shows the scenario where path is consisting of some part of obstacle (b) showing final path after applying segmentRepair operator

\section{SIMULATION RESULTS}

The simulation is performed in $\mathrm{C}$ language and the results have been taken out in the grid environment where user can mark the source and destination positions. The user is also free to mark the static obstacles and dynamic obstacles. The simulation results shown in figure 4 are taken under the same grid size with source and destination positions and population size similar in each case. The figure 5 shows that if the population size increases the proposed algorithm can give better results as compared to original bee colony algorithm. In this case, the source position, destination position and the number of obstacles have been kept same.

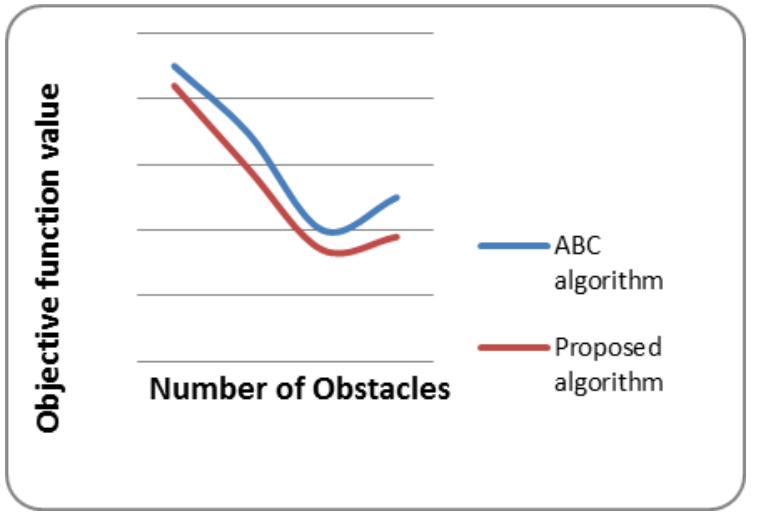

Figure 4: Comparison between $\mathrm{ABC}$ algorithm and proposed algorithm on the basis of number of obstacles

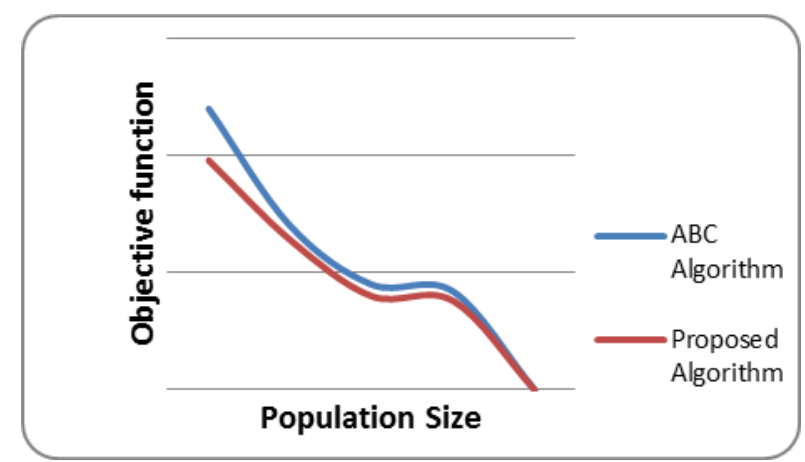

Figure 5: Comparison between $\mathrm{ABC}$ algorithm and proposed algorithm on the basis of population size

\section{CONCLUSION}

The proposed work has been implemented in dynamic environment and the original Artificial Bee Colony Algorithm has been hybridized with two operators so as to improve the final paths obtained. The simulation results have shown that the proposed algorithm generates shorter paths even in the presence of many obstacles. Moreover, if the population size is increased then better results can be obtained as compared to original bee colony algorithm.

\section{REFERENCES}

[1] Buniyamin N., Wan Ngah W.A.J., Sariff N., Mohamad Z., "A Simple Local Path Planning Algorithm for Autonomous Mobile Robots", International Journal Of Systems Applications,Engineering \& Development, Issue 2, Volume 5, 2011

[2] Ananya Das, Priyadarshini Mohapatra, Prakrti Mishra, Pradipta K.Das and Subash C.Mandhata, "Improved real time A* algorithm for Path Planning of Mobile Robot in Quadrant Based Environment", International Journal on Advanced Computer Theory and Engineering (IJACTE), Volume-1, Issue-1, 2012

[3] Santiago Garrido, Luis Moreno, Dolores Blanco and Piotr Jurewicz - Path Planning for Mobile Robot Navigation using Voronoi Diagram and Fast Marching\|, International Journal of Robotics and Automation (IJRA), Volume (2) : Issue (1) : 2011

[4] Pradipta kumar Das, Amit Konar, Romesh Laishram, -Path Planning of Mobile Robot in Unknown Environmentl, Special Issue of IJCCT Vol.1 Issue 2, 3, 4; 
2010 for International Conference [ACCTA-2010], 3-5 August 2010

[5] O. Hachour, "The proposed path finding strategy in static unknown environments", INTERNATIONAL JOURNAL OF SYSTEMS APPLICATIONS, ENGINEERING \& DEVELOPMENT Issue 4, Volume 3,2009

[6] Luciano C. A. Pimenta, Alexandre R. Fonseca, Guilherme A. S. Pereira, Renato C. Mesquita, Elson J. Silva, Walmir M. Caminhas and Mario F. M. Campos, -Robot Navigation Based on Electrostatic Field Computationl, IEEE TRANSACTIONS ON MAGNETICS, VOL. 42, NO. 4, APRIL 2006

[7] Danny Z. Chen, Robert J. Szczerba, and John J. Uhran, Jr.,"A Framed-Quadtree Approach for Determining Euclidean Shortest Paths in a 2-D Environment", IEEE TRANSACTIONS ON ROBOTICS AND AUTOMATION, VOL. 13, NO. 5, OCTOBER 1997

[8] Yi Shen and Mingxin Yuan, -A Novel Poly-clone Particle Swarm Optimization Algorithm and Its Application in Mobile Robot Path Planning\|, Chinese Control and Decision Conference 2010

[9] Song-Hiang Chia, Kuo-Lan Su, Jr-Hung Guo and ChengYun Chung, "Ant Colony System Based Mobile Robot Path Planning", 2010 Fourth International Conference on Genetic and Evolutionary Computing

[10] M.H.Saffari and M.J. Mahjoob, "Bee Colony Algorithm for Real -Time Optimal Path Planning of Mobile Robots", IEEE 2009
[11] Gihan NAGIB, W. GHARIEB, "PATH PLANNING FOR A MOBILE ROBOTUSING GENETIC ALGORITHMS”, IEEE 2004

[12] Jiann-Horng Lin and Li-Ren Huang, "Chaotic Bee Swarm Optimization Algorithm for Path Planning of Mobile Robots", Proceedings of the 10th WSEAS International Conference on EVOLUTIONARY COMPUTING

[13] P.V.Savsani, R.L.Jhala, —Optimal Path Planning for a Robot Arm by using Artificial Bee Colony(ABC) Algorithmll, International Journal of Modern Engineering Research(IJMER) , Volume No.2, Issue 5 Sep-Oct 2012,pp 4434-4438

[14] Joon-Woo Lee, Byoung-Suk Choi, Kyoung-Taik Park and Ju-Jang Lee, "Comparison between Heterogeneous Ant Colony Optimization Algorithm and Genetic Algorithm for Global Path Planning of Mobile Robot", IEEE 2011

[15] Li gao-liang, Shi xu-hua," An improved IGA based path planning of mobile robot in dynamic environment", 2011 Seventh International Conference on Natural Computation

[16] Christos Alexopoulos and Paul M. Griffin, "Path planning for Mobile Robot",IEEE Transactions for Man, System and Cybernetics, Volume 22, No.2, March/April 1992

[17] G Pampara and AP Engelbrecht, "Binary Artificial Bee Colony Optimization”, IEEE 2011 